\title{
BMJ Open Interventions to reduce stigma towards mental disorders in young people: protocol for a systematic review and meta-analysis
}

\author{
Daniel Núñez (D) , ,2 Pablo Martínez (D) , ${ }^{2,3,4}$ Francesca Borghero (D) ,2 \\ Susana Campos (D) , ${ }^{1,2}$ Vania Martínez (D) 2,4,5
}

To cite: Núñez D, Martínez $P$, Borghero $\mathrm{F}$, et al. Interventions to reduce stigma towards mental disorders in young people: protocol for a systematic review and meta-analysis. BMJ Open 2021;11:e045726. doi:10.1136/ bmjopen-2020-045726

- Prepublication history and additional supplemental material for this paper are available online. To view these files, please visit the journal online (http://dx.doi.org/10.1136/ bmjopen-2020-045726)

Received 16 October 2020 Accepted 30 October 2021

Check for updates

(C) Author(s) (or their employer(s)) 2021. Re-use permitted under CC BY-NC. No commercial re-use. See rights and permissions. Published by BMJ.

For numbered affiliations see end of article.

Correspondence to Mr Pablo Martínez; pablo.martinezd@usach.cl

\section{ABSTRACT}

Introduction The stigma towards mental disorders can limit the use and effectiveness of available mental health interventions for young people. We aim to systematically review effectiveness of interventions to reduce stigma towards mental disorders in young people, as evidence has not been recently and systematically synthesised on this topic. Methods and analysis We will conduct a systematic review and meta-analysis of randomised or controlled clinical trials of interventions to reduce stigma towards mental disorders in people aged 10-24 years. Studies involving a comparison group, post intervention and/ or follow-up assessments of knowledge, attitudes and/ or behaviours towards mental disorders (including helpseeking behaviours), will be included. The Cochrane Central Register of Controlled Trials (CENTRAL), Cumulative Index to Nursing and Allied Health Literature (CINAHL), Embase, PubMed and PsycINFO databases will be searched, without time limits, for eligible studies in English or Spanish, and with results available. Databases will be searched from July 2020 to April 2021. The study selection process, the data extraction and the critical evaluationwith the Cochrane risk-of-bias tool-of included studies will be performed independently and in duplicate by teams of reviewers, with the assistance of a third party, until reaching a high degree of agreement. In the presence of substantial heterogeneity $\left(1^{2}>75 \%\right)$, a narrative synthesis of the study results will be used. If feasible, we will also conduct a quality effects model for the statistical synthesis of results. If sufficient data are available, subgroup analyses will be performed to assess potential sources of heterogeneity. Doi plots and the Luis Furuya-Kanamori index will be used to assess publication bias. The Grades of Recommendation, Assessment, Development and Evaluation approach will be used to assess the confidence in the evidence reviewed.

Ethics and dissemination Results are expected to be published in a peer-reviewed journal in the field of adolescent and/or youth mental health.

PROSPERO registration number CRD42020210901.

\section{INTRODUCTION}

Mental health problems in adolescents and young adults are prevalent yet undertreated. ${ }^{12}$ The pooled prevalence for mental
Strengths and limitations of this study

- This protocol for systematic review and metaanalysis has been prepared in accordance with the Preferred Reporting Items for Systematic Review and Meta-Analysis Protocols.

- The Cochrane Handbook for Systematic Reviews of Interventions was used to assist the design of this systematic review.

- There are no recent syntheses of the evidence on interventions to reduce stigma towards mental disorders, a relevant aspect influencing the access and effectiveness of mental health services for young people.

- This systematic review will be limited to published articles.

- Searches will be limited to studies published in English and Spanish languages.

disorders between the ages of 10 and 24 has been estimated at $14.4 \%$, with the subgroups of anxiety disorders $(4.1 \%)$ and depressive disorders $(2.5 \%)$ presenting the highest frequency. ${ }^{3}$ Mental disorders are associated with long-term disability and poor functioning in adulthood, ${ }^{45}$ being linked to poor performance in school and unemployment. ${ }^{5} 6$ Additionally, they are risk factors for psychosocial outcomes such as criminality, self-destructive behaviours, substance abuse and chronic disease. ${ }^{457}$

In a synthesis of 38 systematic reviews, Das et $a l^{8}$ identified effective psychosocial interventions along the continuum of care to improve mental health in adolescents and young adults. Many of these are acceptable and relatively affordable for young people and their families. ${ }^{8}$ Some of them involve educational communities, such as life skills programmes and depression and suicide prevention programmes. These interventions seem to be more accessible and acceptable than traditional mental healthcare, and show 
positive effects on self-esteem, self-efficacy and the reduction of depressive symptoms. ${ }^{8}$

Nevertheless, there are major gaps in access to treatment for mental health problems in young people. ${ }^{9}$ Morris $e t a l^{10}$ evaluated the characteristics and capacities of mental health services for individuals less than 18 years of age in 42 low-income and middle-income countries and found that standard mental health services, lacking properly trained and/or specialised personnel, offer a limited range of benefits for this age group. Moreover, the help-seeking process in this population is further hindered by the stigma towards mental health problems in young people. ${ }^{11} 12$

Stigma towards mental disorders is one of the main barriers for help-seeking and access to mental health services among people of all ages. ${ }^{13}{ }^{14}$ Mental health stigma, whether public or internalised, comprises deeply disparaging attributes involving stereotypes, prejudices and discrimination towards mental disorders. ${ }^{12}{ }^{13}$ These features can result in fear, social exclusion, and authoritarian and paternalistic attitudes, representing serious threats for self-esteem, self-efficacy, and interpersonal relationships in people with mental disorders. ${ }^{13}$

Rüsch et $a l^{13}$ reported the use of three strategies to reduce stigma: concerted protest actions against public statements, media reports and stigmatising advertisements about mental health; education to provide information contradictory to stigma, through various means (eg, books or videos); and contact with people with mental disorders to reduce stereotypes and stigma towards mental health problems. Additionally, Thornicroft $e t a l^{15}$ in a narrative literature review, state that educational interventions either combined with direct contact or not, have received the most scholarly attention. This type of intervention also displays the most evidence of effectiveness in improving knowledge and attitudes towards mental health. However, the evidence about behavioural changes is scarce and inconclusive. ${ }^{15}$

Interventions to reduce mental health-related stigma and discrimination have been tested in multiple population groups, including young people in educational institutions; however, the evidence regarding school-based interventions is limited. ${ }^{15}$ Only two clinical trials were reported in a previous systematic review which included studies of doubtful methodological quality. ${ }^{16}$ Another systematic review of antistigma interventions in university students found a greater number of studies, ${ }^{17}$ although with major methodological limitations. ${ }^{15}$ In both cases, results suggest that these interventions have an effect on knowledge and attitudes in the short term, without further evidence of their impact on behaviour. ${ }^{15}$

The most recent research on stigma in non-adult populations has focused on the following aspects: (1) sociocultural processes involved in the development of mental health stigma at early ages ${ }^{18}$; (2) contributing factors to the stigma towards mental health problems in children and adolescents ${ }^{11}{ }^{12}$; and (3) the potential stigmatising effect of selective interventions in mental health in educational contexts. ${ }^{19}$ The only relatively recent integrative report assessing the effectiveness of interventions to reduce stigma towards mental disorders in young people focused on the process of implementing an antistigma programme in Canadian schools. However, it failed to report the evidence in a systematic way. ${ }^{20}$

Considering that (1) a growing number of interventions have shown to be effective in the management of mental disorders in young people, many of which are based on educational institutions, ${ }^{8}$ that (2) the stigma towards mental disorders can limit the use and effectiveness of these interventions and that (3) this may prolong the suffering of those affected, this systematic review and meta-analysis will synthesise the evidence on effectiveness of interventions to reduce stigma towards mental disorders in young people.

\section{METHODS}

This protocol for systematic review and meta-analysis has been prepared in accordance with the Preferred Reporting Items for Systematic Review and Meta-Analysis Protocols guidelines. ${ }^{21}$

\section{Eligibility criteria}

We will include studies reported in Spanish or English, with no date limit for indexing in the databases described in the Sources of information section. Study protocols or reports that do not include study results will be excluded. The study eligibility criteria defined are presented below:

\section{Participants}

Young people-defined as individuals between 10 and 24 years of age-their parents and/or caregivers and educational/health professionals. Studies with participants outside of this age range will be included as long as the sample mean or median is within the range.

For studies conducted with individuals between 10 and 12 years of age, we will identify those using parental/ teacher reports for determination of psychopathology and stigma. Additionally, if data allow, we will conduct sensitivity analysis evaluating whether effectiveness of interventions vary per age groups, with due attention to studies conducted in individuals aged 10-12 years.

The studies reviewed may include participants with or without mental disorders. However, because it is important to ensure that mental disorders-when present-are adequately assessed, we will report the methods and procedures through which participants are defined as such (eg, application of validated self-report questionnaires), diagnostic evaluation by a professional psychologist or psychiatrist (eg, clinical interview or semistructured interview for these purposes) and/or review of clinical records.

\section{Interventions}

Interventions involving an active component to reduce stigma towards mental disorders in young people. These 
interventions will consider a broad definition of stigma, such as public stigma, structural discrimination, interpersonal stigma (Link et al, 2001) ${ }^{22}$ and self-stigma (Rüsch et al, 2005) ${ }^{13}$ Interventions will be classified as follows ${ }^{17}$ : (1) social contact; (2) video-based social contact; (3) video-based education; (4) education-conference; (5) education-text reading; (6) famous movies; (7) education-role play; and (8) others. The interventions considered may be delivered individually or in groups; at home, in the community (eg, schools, universities and work places), or in health facilities; and using face to face, online, or blended approaches. No limits on intervention duration and/or periodicity were set. The interventions reviewed may be delivered by laymen, educators, health personnel or mental health specialists, among others.

\section{Comparators}

Comparisons with active components (ie, studies comparing two antistigma interventions) improved standard treatment, standard treatment, waiting list, placebo or no intervention. Studies without a comparison group will be excluded.

\section{Outcomes}

Primary outcomes will include information from post intervention and/or follow-up evaluations of knowledge, attitudes and/or behaviours (ie, stereotypes, prejudices and/or discriminatory behaviours) towards mental disorders, as well as help-seeking behaviours or access to mental health services. Secondary outcomes will include self-efficacy, self-esteem and/or empowerment, specifically in young people with mental health problems. Outcome measures related to sociooccupational/educational spheres (eg, work and study situation, and relationship status) will be included.

We will conduct sensitivity analyses based on whether outcomes were assessed using validated versus nonvalidated instruments. We will restrict these analyses to the primary outcomes.

\section{Study design}

Randomised clinical trials and controlled clinical trials (ie, where randomisation has not been explicitly reported, but cannot be ruled out) will be included. Systematic reviews and/or meta-analyses of stigma towards mental disorders in young people will be included in order to find eligible primary studies.

\section{Information sources}

The following databases will be searched from July 2020 up to April 2021: The Cochrane Central Register of Controlled Trials (CENTRAL), Cumulative Index to Nursing and Allied Health Literature (CINAHL), Embase, PubMed, and PsycINFO. In addition, the reference lists of the selected articles will be explored.

The review will be focused on high quality peer-reviewed articles, and grey literature will be excluded. The possible publication biases will be discussed as a limitation of the study.

\section{Search strategy}

The basic search strategy will combine index terms and free-text terms for the following concepts: 'stigma', 'mental health', 'mental disorder', 'adolescents' and 'youths', all of them considered synonyms. Additionally, terms relative to the study design (ie, 'randomised controlled trial') will also be included in the search. The search strategies for each database are detailed in .

We will evaluate both precision and recall values of the search strategy. Precision is the number of included references retrieved by a certain database divided by the total number of search results retrieved by that database. Recall is the number of included references found in the search results for the original search strategies for a database divided by the total number of included references retrieved by all databases together.

\section{Data management}

The review is conducted by four trained researchers (three psychologists and one psychiatrist) having expertise in evidence syntheses, psychopathology, and child and adolescent mental health. The reviewers have followed the recommendations stated in the Cochrane Handbook for Systematic Reviews of Interventions, and have discussed and agreed on the use of standardised data extraction forms suggested by the Cochrane Collaboration (https://dplp. cochrane.org/data-extraction-forms).

Moreover, the data extraction process is being conducted in accordance with known recommendations in the fieldfor example, independent and duplicate data extractionreliability assessments are done by jointly reviewing and discussing extracted data, a process made to ensure quality and appropriateness of gathered information.

One of the reviewers, with experience in database management, will perform the searches and consolidate the search results. After identifying the records in the databases and removing duplicates, the study selection process (from reviewing titles and abstracts to fulltext evaluation) will be carried out independently by four reviewers, who will refer to a third party in case of disagreement. The same procedure will be carried out for coding and extracting the data in the studies. This procedure will consist in conducting searches and consolidating multiple reports from the same study to avoid duplicating data, and then extracting substantive information for the systematic review. The data extraction procedure will incorporate study identification (authors, year of publication and country), characteristics of the participants (eg, age range, sex, presence and type of mental disorders), interventions and comparators (eg, periodicity, duration, format, content, target population and type of stigma addressed), outcomes and study design. The selection and data extraction will consider a calibration phase, where an initial number of studies will be randomly selected and iteratively evaluated until a high level of agreement is reached among the reviewers. This process guarantees the homogeneity of criteria. 
Regarding the extraction of results from the individual studies, the measures for summarising results will be recorded for continuous or dichotomous outcomes in each group (ie, intervention and comparator) used in the primary studies. This includes their respective measures of dispersion (eg, CIs), identifying short-term (up to 3 months post intervention), medium-term (up to 6 months post intervention) and long-term (greater than 6 months post intervention) outcomes.

\section{Critical evaluation of included studies}

The Cochrane tool will be administered to assess the risk of selection, performance, detection, attrition and notification bias in each of the included studies. Risk of bias will be classified as 'high', 'low' or 'unclear'. ${ }^{23}$ In the latter case, reviewers will refine their evaluation criteria, analysing independently and in duplicate a random subset of studies until reaching a high degree of agreement. Thereafter, they will independently review the rest of the studies without duplicates. These results will be discussed among the reviewers, who will examine verbatim excerpts of reports of the selected studies, included to support the reviewers' judgement.

\section{Synthesis of included studies}

A narrative synthesis, accompanied by comparative tables, will be used to characterise the studies included in the systematic review, along with their critical evaluation. Even though the presence of substantial clinical and methodological variety is anticipated, which may make the statistical synthesis of the results of the selected studies impractical (ie, meta-analysis), formal analyses of heterogeneity will be performed by exploring forest plots and using the $\mathrm{I}^{2}$ statistic to determine the consistency of the results of studies in meta-analyses. ${ }^{24}$ In case of substantial heterogeneity (ie, inconsistent results in the forest plots and/or $\mathrm{I}^{2}>75 \%$ ), a narrative synthesis of results will be decided on, accompanied by comparative tables of measures to summarise results in individual studies, considering short-term, medium-term and longterm outcomes. If the performance of a meta-analysis is feasible, then, considering recent developments on this technique, a meta-analysis of heterogeneous studies will be conducted using the quality effects estimator, which gives greater weight to large and better-quality studies. ${ }^{25}$ If sufficient data are available, subgroup analyses will be performed to assess potential sources of heterogeneity (eg, age subgroups, presence of mental disorders, types of interventions). Statistical analyses will be performed with MetaXL, a free Excel plugin for meta-analysis. ${ }^{26}$

\section{Metabiases}

The appraisal of selectively reported outcomes can be a source of bias in the individual studies; therefore, the procedures recommended by the Cochrane tool for the evaluation of bias risk will be used. ${ }^{23}$ Thus, the study protocols of the randomised or controlled clinical trials will be reviewed to establish if the results of the outcomes specified in the protocol were reported. If discrepancies are found, a high risk of bias for this dimension will be assumed.

If meta-analyses are performed, Doi plots will be used and explored along with the Luis Furuya-Kanamori index to assess the presence of asymmetry attributable to publication bias in the effects of the studies. ${ }^{27}$

\section{Confidence in estimates of effect}

In accordance with the Cochrane Collaboration recommendations, the Grades of Recommendation, Assessment, Development and Evaluation approach will be used for an assessment that comprehensively and systematically considers the risk of bias between studies, inconsistency between effect estimates, inaccuracy of results, lack of evidence directionality, publication bias and factors that may increase confidence in the effect (ie, large effects). ${ }^{23}$

\section{Patient and public involvement}

This is a research carried out without patients or members of the public. Patients did not participate in the design of the protocol.

\section{Author affiliations}

${ }^{1}$ Associative Research Program, Center on Cognitive Sciences, Faculty of Psychology, Universidad de Talca, Talca, Chile

${ }^{2}$ Millennium Nucleus to Improve the Mental Health of Adolescents and Youths, Imhay, Santiago, Chile

${ }^{3}$ Faculté de médecine et des sciences de la santé, Université de Sherbrooke, Longueuil, Quebec, Canada

${ }^{4}$ Millennium Institute for Research inDepression and Personality (MIDAP), Santiago, Chile

${ }^{5}$ Centro de Medicina Reproductiva y Desarrollo Integral del Adolescente (CEMERA), Facultad de Medicina, Universidad de Chile, Santiago, Chile

Contributors PM designed the study protocol and wrote the manuscript. DN, SC, $\mathrm{FB}$ and VM wrote the manuscript, reviewed and revised critically it.

Funding This work was funded by ANID - Millennium Science Initiative Program - NCS17_035 and by the Programa de Investigación Asociativa (PIA) en Ciencias Cognitivas, Facultad de Psicología, Universidad de Talca. VM received funding from ANID, ICS13_005.

Disclaimer The funders/sponsors had no role in the design of the study protocol or decision to submit the study protocol for publication.

Competing interests None declared.

Patient and public involvement statement This is a research carried out without patients or members of the public. Patients did not participate in the design of the protocol.

\section{Patient consent for publication Not applicable.}

Ethics approval This study was conducted using published data and did not involve human participants directly. Therefore, it is exempt of ethical review in this regard. Results are expected to be published in a peer-reviewed journal in the field of mental health in adolescent and/or youth populations.

Provenance and peer review Not commissioned; externally peer reviewed.

Supplemental material This content has been supplied by the author(s). It has not been vetted by BMJ Publishing Group Limited (BMJ) and may not have been peer-reviewed. Any opinions or recommendations discussed are solely those of the author(s) and are not endorsed by BMJ. BMJ disclaims all liability and responsibility arising from any reliance placed on the content. Where the content includes any translated material, BMJ does not warrant the accuracy and reliability of the translations (including but not limited to local regulations, clinical guidelines, terminology, drug names and drug dosages), and is not responsible for any error and/or omissions arising from translation and adaptation or otherwise.

Open access This is an open access article distributed in accordance with the Creative Commons Attribution Non Commercial (CC BY-NC 4.0) license, which 
permits others to distribute, remix, adapt, build upon this work non-commercially, and license their derivative works on different terms, provided the original work is properly cited, appropriate credit is given, any changes made indicated, and the use is non-commercial. See: http://creativecommons.org/licenses/by-nc/4.0/.

\section{ORCID iDs}

Daniel Núñez http://orcid.org/0000-0001-7091-2249

Pablo Martínez http://orcid.org/0000-0003-4482-7993

Francesca Borghero http://orcid.org/0000-0002-4304-2353

Susana Campos http://orcid.org/0000-0002-9455-2695

Vania Martínez http://orcid.org/0000-0001-5980-7122

\section{REFERENCES}

1 Kessler RC, Angermeyer M, Anthony JC, et al. Lifetime prevalence and age-of-onset distributions of mental disorders in the world Health organization's world mental health survey initiative. World Psychiatry 2007;6:168-76.

2 Silva SA, Silva SU, Ronca DB, et al. Common mental disorders prevalence in adolescents: a systematic review and meta-analyses. PLoS One 2020;15:e0232007.

3 Institute for Health Metrics and Evaluation (IHME). GBD Results Tool. Seattle, WA: IHME, University of Washington, 2020http://ghdx. healthdata.org/gbd-results-tool

4 Costello EJ, Maughan B. Annual research review: optimal outcomes of child and adolescent mental illness. J Child Psychol Psychiatry 2015;56:324-41.

5 Erskine HE, Norman RE, Ferrari AJ, et al. Long-Term Outcomes of Attention-Deficit/Hyperactivity Disorder and Conduct Disorder: A Systematic Review and Meta-Analysis. J Am Acad Child Adolesc Psychiatry 2016;55:841-50.

6 Clayborne ZM, Varin M, Colman I. Systematic review and metaanalysis: adolescent depression and long-term psychosocial outcomes. J Am Acad Child Adolesc Psychiatry 2019;58:72-9.

7 Groenman AP, Janssen TWP, Oosterlaan J. Childhood psychiatric disorders as risk factor for subsequent substance abuse: a metaanalysis. J Am Acad Child Adolesc Psychiatry 2017:56:556-69.

8 Das JK, Salam RA, Lassi ZS, et al. Interventions for adolescent mental health: an overview of systematic reviews. Journal of Adolescent Health 2016;59:S49-60.

9 Radez J, Reardon T, Creswell C, et al. Why do children and adolescents (not) seek and access professional help for their mental health problems? A systematic review of quantitative and qualitative studies. Eur Child Adolesc Psychiatry 2021;30:183-211.

10 Morris J, Belfer M, Daniels A, et al. Treated prevalence of and mental health services received by children and adolescents in 42 low-and-middle-income countries. J Child Psychol Psychiatry 2011;52:1239-46.

11 Aguirre A, Cruz I, Billings J. What are the barriers, facilitators and interventions targeting help-seeking behaviours for common mental health problems in adolescents? A systematic review. BMC Psychiatry 2020;20.

12 Kaushik A, Kostaki E, Kyriakopoulos M. The stigma of mental illness in children and adolescents: a systematic review. Psychiatry Res 2016;243:469-94.

13 Rüsch N, Angermeyer MC, Corrigan PW. Mental illness stigma: concepts, consequences, and initiatives to reduce stigma. Eur Psychiatry 2005;20:529-39.

14 Schnyder N, Panczak R, Groth N, et al. Association between mental health-related stigma and active help-seeking: systematic review and meta-analysis. Br J Psychiatry 2017;210:261-8.

15 Thornicroft G, Mehta N, Clement S, et al. Evidence for effective interventions to reduce mental-health-related stigma and discrimination. Lancet 2016;387:1123-32.

16 Schachter HM, Girardi A, Ly M, et al. Effects of school-based interventions on mental health stigmatization: a systematic review. Child Adolesc Psychiatry Ment Health 2008;2:18 https://dx.doi.org/ 10.1186\%2F1753-2000-2-18

17 Yamaguchi S, Wu S-I, Biswas M, et al. Effects of short-term interventions to reduce mental Health-Related stigma in University or college students. Journal of Nervous \& Mental Disease 2013;201:490-503.

18 Mueller J, Callanan MM, Greenwood K. Communications to children about mental illness and their role in stigma development: an integrative review. J Ment Health 2016;25:62-70.

19 Gronholm PC, Nye E, Michelson D. Stigma related to targeted school-based mental health interventions: a systematic review of qualitative evidence. J Affect Disord 2018;240:17-26.

20 Chen SP, Sargent E, Stuart H. Effectiveness of school-based interventions on mental health stigmatization. In: Leschied AW, Saklofske DH, Flett GL, eds. Handbook of school-based mental health promotion: an evidence-informed framework for implementation. New York, NY: Springer., 2018: 201-12.

21 Moher D, Shamseer L, Clarke M, et al. Preferred reporting items for systematic review and meta-analysis protocols (PRISMA-P) 2015 statement. Syst Rev 2015;4:1.

22 Link BG, Phelan JC. Conceptualizing stigma. Annu Rev Sociol 2001;27:363-85.

23 Higgins JPT, Green S. Cochrane handbook for systematic reviews of interventions. Cochrane Book Series. The Cochrane Collaboration(editors). (2017https://handbook-5-1.cochrane.org/

24 Higgins JPT, Thompson SG, Deeks JJ, et al. Measuring inconsistency in meta-analyses. BMJ 2003;327:557-60.

25 Doi SAR, Barendregt JJ, Khan S, et al. Advances in the metaanalysis of heterogeneous clinical trials II: the quality effects model. Contemp Clin Trials 2015;45:123-9.

26 Barendregt JJ, Doi SA. MetaXL User Guide version 5.3. EpiGear International Pty Ltd. Queensland, Australia., 2016http://www. epigear.com/index_files/MetaXL\%20User\%20Guide.pdf

27 Furuya-Kanamori L, Barendregt JJ, Doi SAR. A new improved graphical and quantitative method for detecting bias in metaanalysis. Int J Evid Based Healthc 2018;16:195-203. 